\title{
Einsatz der Brennstoffzelle als Netzersatzanlage sicherheitsrelevanter Digitalfunkstationen im Feldtest
}

\author{
Denny Ragusch*, Michael Jergović, Lutz B. Giese und Siegfried Rolle
}

\section{Zusammenfassung}

Es wird anhand des Förderprojekts „Wasserstoff-Netzersatzanlagen der Behörden und Organisationen mit Sicherheitsaufgaben (BOS) des Landes Brandenburg" des Zentraldienstes der Polizei des Landes Brandenburg die Praxistauglichkeit von Brennstoffzellen-Netzersatzanlagen untersucht. Marktreife und kommerziell verfügbare Brennstoffzellen wurden in 115 sicherheitsrelevanten Digitalfunkstationen im Flächenland Brandenburg als Ersatz von wartungsintensiven und umweltproblematischen Dieselgeneratoren für die (Not-)Stromversorgung installiert. Diese Netzersatzanlagen sind für eine Überbrückungszeit von $72 \mathrm{~h}$ unter realen Bedingungen ausgelegt und wurden im Feldtest, parallel zum realen Betrieb, über einen längeren Einsatzzeitraum (ca. 2 Jahre) auf ihre Zuverlässigkeit und Verfügbarkeit hin untersucht. Die Anforderungen an und die Realisierung der Brennstoffzellen-Netzersatzanlagen sowie die Feldtest-Versuchsplanung und deren Ergebnisse werden beschrieben und diskutiert. Die Praxistauglichkeit wird mit einer hohen Verfügbarkeit über $97 \%$ und einer Funktionszuverlässigkeit von mehr als $95 \%$ erfolgreich bestätigt. Die Entwicklung weiterer Komponenten wird empfohlen.

\begin{abstract}
On the basis of the funding project "Hydrogen Based Backup Power Systems of Public Authorities and Organizations with Security Tasks (BOS) of the German Federal State of Brandenburg" of the Central Police's Services of the German Federal State of Brandenburg, the practicability of fuel-cell backup power systems is investigated. Within this project, market-ready and commercially available fuel-cell systems were installed in 115 security-relevant digital telecommunication units in the Federal State of Brandenburg as a replacement for maintenance-intensive and environmentally-hazardous diesel generators for (emergency) power supply. These emergency power systems are designed for a bridging time of 72 hours under real conditions and have been tested for their reliability and availability over a longer period of operation (approx. 2 years) in the field test, simultaneously with real operation. The requirements and realization of the fuel cell backup power systems as well as the field test planning and their results are described and discussed. The practicability is successfully confirmed with a high availability of more than $97 \%$ and a functional reliability of more than $95 \%$. Additional development requirements for further components are recommended.
\end{abstract}

\section{Einführung und Zielstellung}

Die konventionelle Netzersatzanlage (NEA) mit Dieselgenerator ist wartungsintensiv (Kurtz et al. 2015) und umweltproblematisch (Gagge 2008), zudem sind langfristig Preissteigerungen des Dieseltreibstoffs infolge der global begrenzten Verfügbarkeit zu erwarten. Eine umweltfreundliche Alternative stellt die BrennstoffzellenNEA (BZ-NEA) dar (Serincan 2016). Sie ist unter anderem wartungsarm, zuverlässig, nahezu geräuschlos im Betrieb und durch einfache Erweiterung des Brennstofflagers flexibel in der Überbrückungszeitdauer (Nordin \&
Lindemark 1999). Die BZ-NEA übernimmt bei einem Stromausfall die Notstromversorgung und ist von der unterbrechungsfreien Stromversorgung (USV) zu unterscheiden. Während der Umschaltung auf den Notstrombetrieb kann es beispielsweise bei der NEA zu einem kurzfristigen Spannungsabfall kommen. Eine USV ist zudem in der Regel nur für wenige Minuten bis einige Stunden Überbrückungszeit ausgelegt, die NEA je nach Anwendungsfall für einen längeren Überbrückungszeitraum. Ziel dieses Beitrags ist es, die Praxistauglichkeit der BZ-NEA zu überprüfen.
Betriebserfahrungen und Feldtestmessdaten wurden im Polizeiprojekt „W-NEA BOS BB - Wasserstoff-Netzersatzanlagen der Behörden und Organisationen mit Sicherheitsaufgaben des Landes Brandenburg" (Förderkennzeichen: 03BS224) im Rahmen des Nationalen Innovationsprogramms „Wasserstoff- und Brennstoffzellentechnologie“ gewonnen. Neben der Durchführung eines Feldtests zur Demonstration der Praxistauglichkeit der BZ-NEA war die Marktvorbereitung ein wesentliches Projektziel. Wissenschaftlich-technisch wurde das Projekt durch die Technische Hochschule Wildau (THWi) begleitet. Der Einsatz 
der Brennstoffzelle erfolgte unter realen Betriebsbedingungen als Netzersatzanlage in sicherheitsrelevanten Digitalfunkstationen der Polizei im Land Brandenburg. Ein solcher Digitalfunkstandort ist beispielhaft in der Abb. 1 gezeigt. Kurzel (2016) bestimmte folgende relevante Parameter für die Praxistauglichkeit: Zuverlässigkeit, Verfügbarkeit, Sicherheit, Wirtschaftlichkeit und Umwelteinfluss. Im Feldtest wurden die Parameter Zuverlässigkeit und Verfügbarkeit untersucht. Die Sicherheit der Anlagen ist gegeben, da nach den entsprechenden gesetzlichen Vorschriften die Anlagen abgenommen wurden. Alle Anlagen wurden natürlich belüftet, so dass keine aufwändige Sicherheitstechnik insbesondere zum Explosionsschutz installiert werden musste. Auf Grund der im Vergleich zur konventionellen Diesel-NEA deutlich geringeren Wartungs- und Betriebskosten für die Brennstoffzellensysteme (Gagge 2008) können trotz höherer Anschaffungskosten die Anlagen wirtschaftlich betrieben werden (Kurtz et al. 2015). Dieselgeneratoren müssen regelmäßig gewartet und betrieben werden, um die Verfügbarkeit

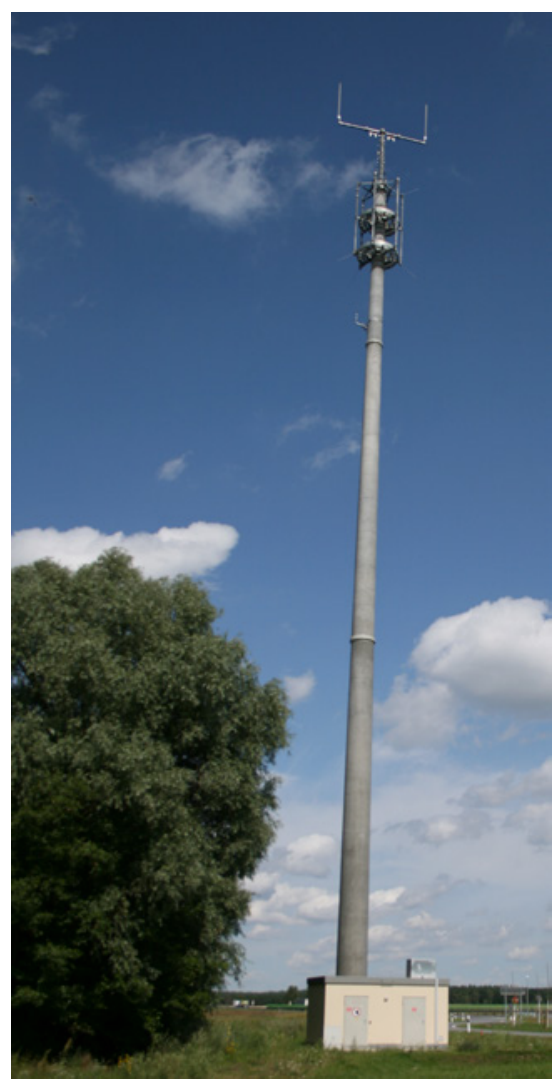

Abb. 1) Foto eines BOS-Digitalfunkmasts mit zugehörigem Funksystemtechnik-Container. Quelle: Projektorganisation Digitalfunk BOS Brandenburg sicherzustellen. Die BZ-NEA wird nur einmal im Jahr gewartet (Gagge 2008). Der Brennstoff kann mittels regenerativ erzeugten Stroms nachhaltig hergestellt werden. Weiterhin kann der Wasserstoff nicht das Erdreich verunreinigen, wie dies bei Diesel-Leckagen möglich ist. Die Abgase bestehen aus unschädlichem Wasserdampf und die Geräuschentwicklung der Brennstoffzellensysteme ist im Vergleich zum Dieselgenerator geringer. Die Praxistauglichkeit der BZ-NEA ist in den Punkten Sicherheit, Wirtschaftlichkeit und Umwelteinfluss somit belegt.

\section{Anforderungen und Beschreibungen der untersuchten Brennstoffzellen- Netzersatzanlagen}

115 Standorte wurden im Feldtest auf drei Anbieter (A, B und C) verteilt. Die wesentlichen Anforderungen an die Brennstoffzellen-Netzersatzanlagen im Feldtest waren:

- geringe Aufstellfläche der NEA samt Treibstofflager

- Vandalismussicherheit

- plausible Service- und Logistikkonzepte

- minimale Überbrückungszeit eines Stromausfalls von $72 \mathrm{~h}$

- Möglichkeit des Brennstoffwechsels während des NEA-Betriebs

- elektrotechnische Einbindung zur vollständigen Ersetzung des Stromnetzes

- Nennleistung für mindestens 2.500 Betriebsstunden

- Aufnahme diverser Umweltmessdaten, wie Lufttemperatur, Luftdruck und Luftfeuchte, für statistischen Auswertungen

- übergreifendes Monitoringsystem

Alle installierten BrennstoffzellenNetzersatzanlagen basierten auf PEM-Brennstoffzellen, die direkt mit Wasserstoff aus Druckgasflaschen betrieben wurden. PEM steht für Proton Exchange Membrane und ist ein häufig eingesetzter Brennstoffzellentyp in vielen Anwendungsbereichen. Für den Betrieb der Brennstoffzellen war ein parallel geschalteter NEA-Akkumulator, insbesondere zum Start der Brennstoffzelle und zur Abdeckung von Leistungsspitzen, notwendig. Die Anbieter setzten entsprechend den gestellten Anforderungen unterschiedliche bauliche Konzepte um. Anbieter A mit einem „Outdoor-Gehäuse“, das alle wesentlichen BZ-NEAKomponenten samt Monitoringsystem enthielt, wird beispielhaft in der Abb. 2 gezeigt. Anbieter $B$ entwickelte eine eigene vandalismussichere Einhausung, die als Anbau an die Funkcontainerwand montiert wurde. Sie enthielt die Wasserstoff-Druckgasflaschen und die Brennstoffzellenmodule. Der BZNEA-Akkumulator, die weiteren elektrotechnischen Komponenten und das Monitoringsystem wurden direkt im Funkcontainer untergebracht. Anbieter $C$ erweiterte den Funkcontainer um einen Anbau, der die wesentlichen BZ-NEA-Komponenten einschließlich Monitoringsystem enthielt.

Die minimale Überbrückungszeit eines Stromausfalls von $72 \mathrm{~h}$ wurde mittels zweier Wasserstoffgebinde mit automatischer Umschaltung, welche unter anderem einen Druckgasflaschentausch während des BZNEA-Betriebs erlaubte, realisiert. Die Flaschenanzahl wurde entsprechend der Systemparameter ausgelegt. Durch die automatische Umschaltung ist es mit entsprechendem Druckgasflaschentausch möglich, die BZ-NEA weit über die $72 \mathrm{~h}$ hinaus kontinuierlich zu betreiben.

Die elektrotechnische Einbindung erfolgte in der Weise, dass bei einem Stromausfall, detektiert durch einen Phasenwächter, nach 15 Minuten die BZ-NEA die Stromversorgung übernahm. Kürzere Stromausfälle wurden von der vorhandenen Funktechnik mit integrierter USV überbrückt. Das EVU-Netz wurde im BZ-NEA-Betrieb durch insgesamt drei redundant ausgeführte Wechselrichter, die aus der Brennstoffzellen-Gleichspannung eine 230 V-Wechselspannung erzeugten, vollständig ersetzt. Eine schematische Darstellung zeigt die Abb. 3. Die elektrische Last ergab sich im Feldtest maßgeblich aus der Funksystemtechnik und der Klimaanlage. Die Klimaanlagenregelung war zum Schutz der Funksystemtechnik so eingestellt, dass die Temperatur im Inneren des Digitalfunkcontainers nicht weniger als $10^{\circ} \mathrm{C}$ und nicht mehr als $32^{\circ} \mathrm{C}$ betrug. Aus vorangegangenen Untersuchungen wurden die erforderlichen Nennleistungen der BZ-NEA bestimmt, die in 
der Tab. 1 mit der Anzahl der untersuchten Anlagen je Anbieter aufgeführt sind. Anbieter $A$ und $C$ verwendeten an allen Standorten ein baugleiches Brennstoffzellensystem mit jeweils zwei FutureE-Jupiter-48-V-Brennstoffzellenmodulen (JU FCU 2.0 48V-23) mit je 2,5 kW maximaler Ausgangsleistung. Hauptbestandteil des Monitoring- und Steuerungssystems der Anbieter A und C war der Heliocentris „Energymanager“. Anbieter B verwendete Brennstoffzellenmodule der Firma ReliOn Inc., für die Nennleistungen „2,5 kW“ und "3 kW“ jeweils ein Brennstoffzellenmodul "ReliOn E-2500“ und ein "ReliOn E-1100“. Bei den Anlagen mit „3,5 kW“ Nennleistung wurden zwei „ReliOn E-2500“ Brennstoffzellenmodule verwendet. Das Monitoring- und Steuerungssystem basierte auf dem "Sitemanager" der GENEREX System $\mathrm{GmbH}$. Hauptaufgabe der Monitoringsysteme im Feld war die Übertragung des aktuellen BZ-NEA-Status, des vorhandenen Brennstoffvorrats mit Abschätzung der minimalen Überbrückungszeit und der System- und Umweltmessdaten sowie die Alarmierung bei Notstrombetrieb. Die Messdaten wurden verschlüsselt über das Mobilfunknetz an die jeweiligen zentralen Datenbankserver der Anbieter gesendet.

\section{Feldtest Versuchsprogramm und Datenauswertung}

Der Feldtest wurde in zwei Betriebsphasen aufgeteilt. Die erste Phase „Betrieb I“ begann direkt nach der erfolgreichen Installation und Inbetriebnahme der jeweiligen BZ-NEA für einen Zeitraum von 24 Wochen. Die Anlagen wurden in dieser Phase wöchentlich entsprechend dem Versuchsprogramm getestet, um Anlagenfehler frühzeitig festzustellen. In der Betriebsphase „Betrieb II“ wurden die Tests monatlich durchgeführt. Alle Tests waren Stromausfallsimulationen, wobei der Testbeginn variierte und bei folgenden Tests jeweils um eine Stunde verschoben wurde. Das öffentliche Netz wurde wie bei einem realen Stromausfall getrennt. Die Dauer der Stromausfallsimulationen wurde wie folgt festgelegt: kurze Stromausfälle dauerten 30 Minuten, lange Stromausfälle 240 Minuten und zwei kurz hintereinander auftretende Stromausfälle je

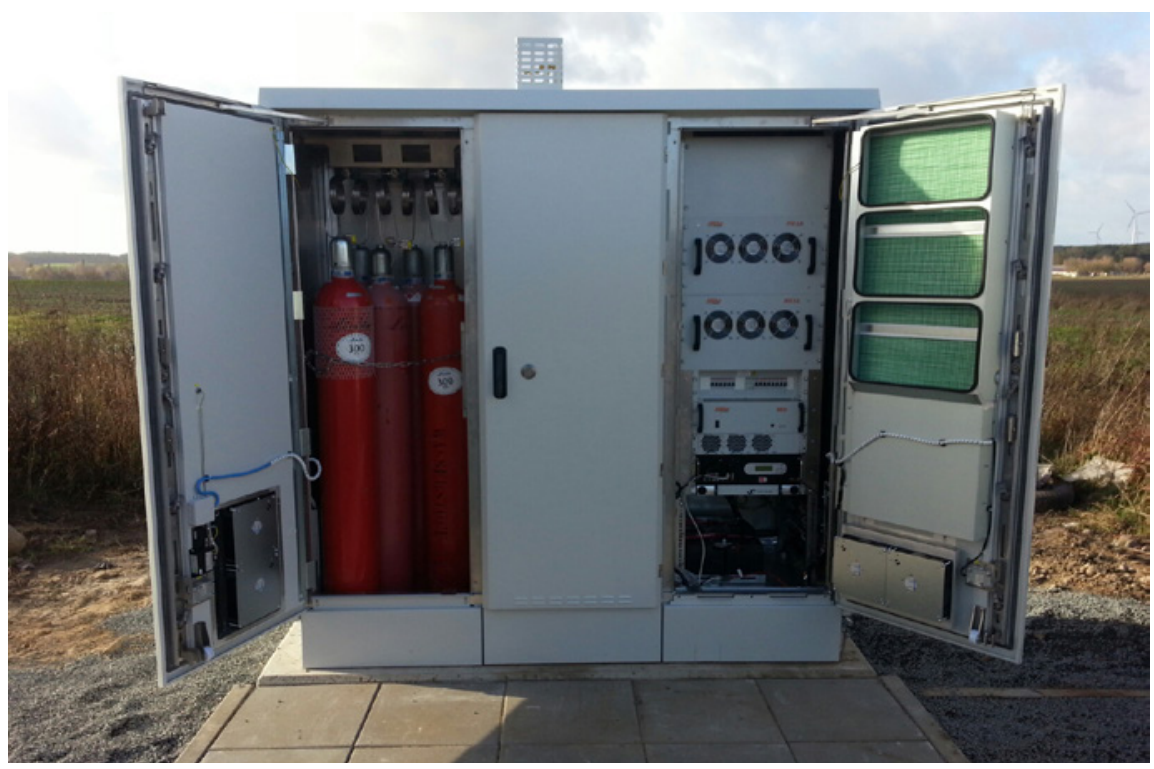

$A b b$. 2) Foto eines geöffneten „Outdoor-Gehäuses“ von Anbieter A; links im Bild sind die Druckgasflaschen in Rot zu erkennen, rechts im Bild die zwei Brennstoffzellenmodule mit Controller, drei Wechselrichter, die Monitoringhardware und der NEA-Akkumulator. Quelle: AdKor $\mathrm{GmbH}$

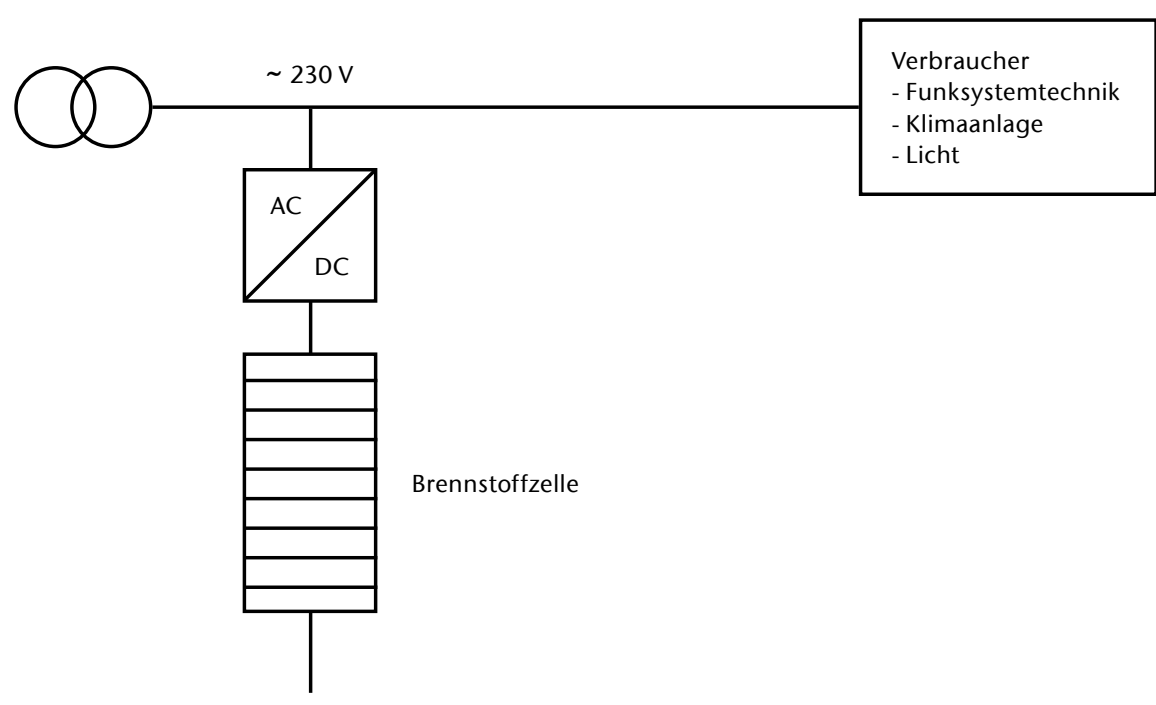

Abb. 3) Schematische Darstellung der elektrotechnischen Einbindung der Netzersatzanlage. Die Netzersatzanlage stellt mittels Wechselrichtern 3-Phasen je $230 \mathrm{~V}$ zur Verfügung.

Tab. 1: Übersicht der Anzahl installierter Brennstoffzellen-Netzersatzanlagen je Anbieter und Nennleistung

\begin{tabular}{|c|c|c|c|}
\hline Nennleistung & \multicolumn{3}{|c|}{ Anzahl der Brennstoffzellen-Netzersatzanlagen-Standorte } \\
\hline & Anbieter A & Anbieter B & Anbieter C \\
\hline $3,5 \mathrm{~kW}$ & 6 & 4 & 3 \\
\hline $3,0 \mathrm{~kW}$ & 18 & 14 & 11 \\
\hline $2,5 \mathrm{~kW}$ & 27 & 21 & 11 \\
\hline Gesamtanzahl: & 51 & 39 & 25 \\
\hline
\end{tabular}


30 Minuten mit 15 Minuten Pause dazwischen. In der Tab. 2 sind die einzelnen Versuchsgruppen aufgeführt. Die Anlagen der Versuchsgruppe V-00 wurden neben den realen Stromausfällen nicht zusätzlich getestet. Die Versuchsgruppen V-00 bis V-03 entsprachen jeweils $10 \%$ der Anlagen je Anbieter. Die Versuchsgruppe V-04 war je Anbieter ein Sonderstandort mit zusätzlicher Messtechnik für weiterführende Untersuchungen. Die Versuchsgruppe V-05 beinhaltete alle restlichen Standorte. Ziel der Versuchsplanung war im Wesentlichen die Bestimmung der Verfügbarkeit und der Zuverlässigkeit, aber auch die Bestimmung des Brennstoffverbrauchs und des Betriebsverhaltens unter besonderen Betriebsbedingungen, wie der Betrieb im Hochsommer oder im Winter.

Die Verfügbarkeit (Arnold 2008: 868) beschreibt die zeitliche Einsatzbereitschaft der Anlagen in Prozent und wird definiert als:

Verfügbarkeit $=\frac{\text { Summe der geplanten Betriebszeiten }- \text { Summe der Ausf allzeiten }}{\text { Summe der geplanten Betriebszeiten }} \cdot 100 \%$

Die Nichtverfügbarkeit eines Brennstoffzellenmoduls und Wartungsarbeiten, bei denen das System nicht verfügbar war, wurden den Ausfallzeiten angerechnet.

Die Betriebszeit der BZ-NEA setzt sich aus funktionsbedingten Wartezeiten (das öffentliche Netz ist vorhanden) und aus ereignisbezogenen Betriebszeiten (der Strom fällt aus) zusammen. Eine ereignisbezogene Funktionszuverlässigkeit (Arnold 2008: 866) ist daher zweckmäßig. Als störungsfreie Funktion wurde der folgende Ablauf definiert:

- Erkennen des Stromausfalls (real oder zu Testzwecken)

- erfolgreiche Notstromversorgung

- fehlerfreier Betrieb der Brennstoffzellenmodule

- erfolgreiches Zurückschalten auf das öffentliche Netz

Funktionszuverlāssigkeit $=\frac{\text { Anzahl Funktionsfähig }}{\text { Anzahl Funktionsfähig }+ \text { Anzahl Funktionsunfähig }} \cdot 100 \%$

Der Brennstoffvorrat je Wasserstoffgebinde wurde durch die Monitoringsysteme näherungsweise mittels des Druck- gasflaschenvolumens und des Wasserstoffdrucks im Gebinde sowie der Außentemperatur bestimmt. Das Wasserstoffgas wurde dabei als ideales Gas angenommen. Da sich der Wasserstoff bei den vorliegenden Betriebsbedingungen nicht wie ein ideales Gas verhält, wurde mittels der Methode nach Zheng et al. (2016) das reale Wasserstoffvolumen bestimmt. Die Abb. 4 verdeutlicht diesen Sachverhalt. Das Wasserstoffvolumen, berechnet unter der Annahme des idealen Gases, weicht bei einer Gastemperatur von $15^{\circ} \mathrm{C}$ und einem Gasdruck im Bereich von 60 bis 300 bar circa von 5 bis zu $20 \%$ vom realen Gas ab. Zur Bestimmung des Wasserstoffverbrauchs wird das gemessene reale Wasserstoffvolumen zweier Zeitpunkte auf Normbedingungen umgerechnet und die Differenz gebildet. Zusammen mit der von den Brennstoffzellen gewandelten elektrischen Energie in dieser Zeitspanne kann der reale Verbrauch in Normliter pro Minute und Kilowatt mit den Herstellerangaben verglichen werden.

\section{Ergebnisse und Diskussion}

In der Tab. 3 sind die zusammengefassten Ergebnisse der Verfügbarkeitsuntersuchung dargestellt. Die mittlere Verfügbarkeit in Prozent aller BZ-NEA betrug während der Projektlaufzeit über $97 \%$. Die Unterschiede der mittleren Verfügbarkeit der nahezu baugleichen Anlagen der Anbieter $A$ und $C$ mit 99,6 \% (A) und 97,4 \% (C) sind durch organisatorische Abläufe zu begründen. Anbieter A erkannte und behob Störungen selbstständig, während Anbieter $C$ vereinbarungsgemäß erst auf die Störungsmeldungen durch den Betreiber reagierte. In den einzelnen Versuchsgruppen sind keine gravierenden Unterschiede der Verfügbarkeit erkennbar. Durch die Behebung vorhandener Softwarefehler bei den Systemen der Anbieter A und C kann die Verfügbarkeit zudem weiter gesteigert werden. Im Wesentlichen sind neben menschlichem Versagen (beispielsweise nicht geöffnete Druckgasflaschen) und den wenigen ausgetauschten Brennstoffzellenmodulen defekte elektrische Umschalteinrichtungen und undichte 300-bar-Wasserstoff-

Tab. 2: Übersicht der Versuchsgruppen mit Stromausfall-Simulationsdauern in den jeweiligen Betriebsphasen.

\begin{tabular}{|c|c|c|c|c|c|c|}
\hline $\begin{array}{l}\text { Woche (Betrieb-I) } \\
\text { Monat (Betrieb-II) }\end{array}$ & V-00 & V-01 & V-02 & V-03 & V-04 & V-05 \\
\hline 1 & - & $30 \mathrm{~min}$ & $30 \mathrm{~min}$ & $30 \mathrm{~min}$ & $240 \mathrm{~min}$ & $30 \mathrm{~min}$ \\
\hline 2 & - & $30 \mathrm{~min}$ & $30 \mathrm{~min}$ & $\begin{array}{c}30 \text { min } \\
15 \text { min Pause } \\
30 \text { min }\end{array}$ & $30 \mathrm{~min}$ & $30 \mathrm{~min}$ \\
\hline 3 & - & $30 \mathrm{~min}$ & $30 \mathrm{~min}$ & $30 \mathrm{~min}$ & $\begin{array}{c}30 \mathrm{~min} \\
15 \text { min Pause } \\
30 \text { min }\end{array}$ & $30 \mathrm{~min}$ \\
\hline 4 & - & $\begin{array}{c}30 \mathrm{~min} \\
15 \mathrm{~min} \text { Pause } \\
30 \mathrm{~min}\end{array}$ & $240 \mathrm{~min}$ & $240 \min$ & $30 \mathrm{~min}$ & $30 \mathrm{~min}$ \\
\hline
\end{tabular}




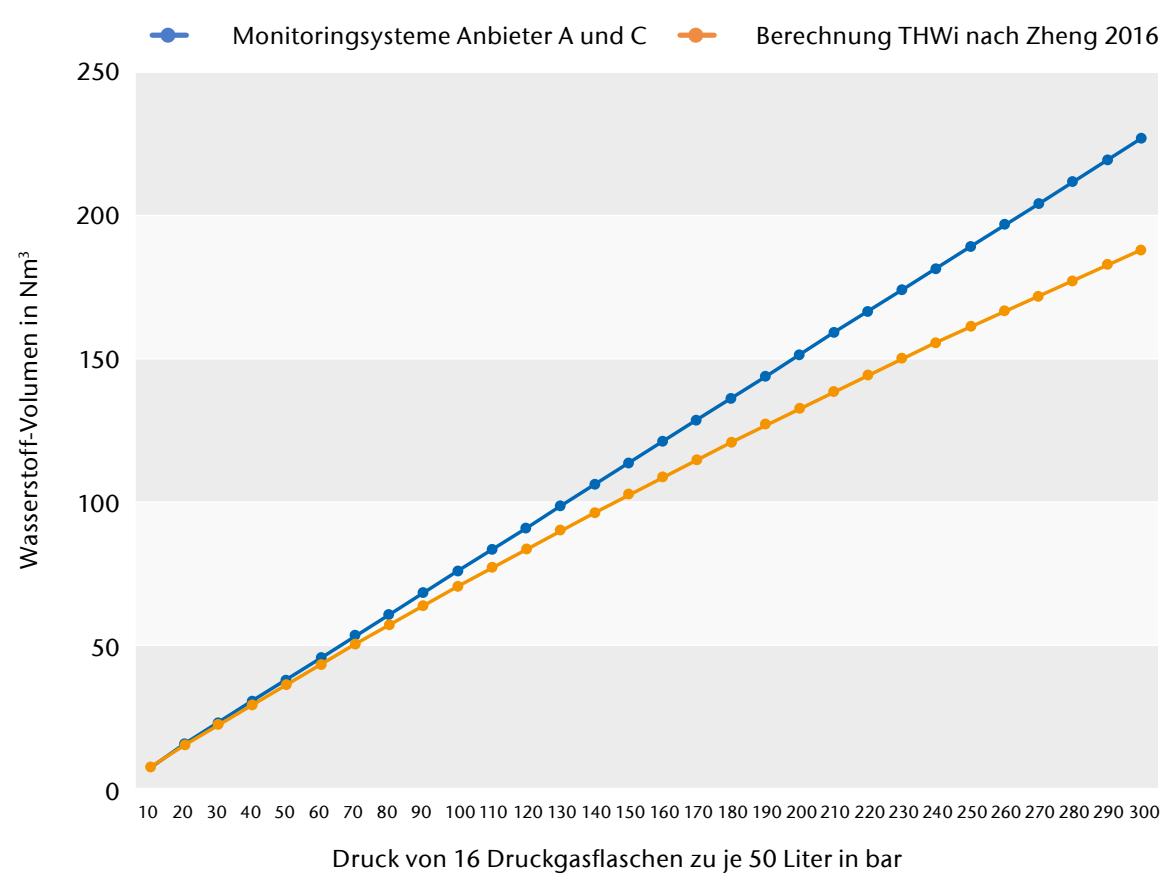

$A b b .4)$ Vergleich der Wasserstoff-Volumenberechnung durch das Monitoringsystem von Anbieter $A$ und $C$ (Annahme ideales Gas) gegenüber der Berechnung durch die THWi (reales Gas) nach Zheng et al. 2016; 16 Druckgasflaschen zu je 50 Liter; Berechnung für eine Gastemperatur von $15^{\circ} \mathrm{C}$.

armaturen verfügbarkeitsmindernd aufgefallen. Alle Monitoringsysteme konnten schleichende Wasserstoffleckagen nicht detektieren. Die Serviceund Logistikkonzepte wurden durch die Logistikexperten der Gruppe von Herrn Prof. Dr.-Ing. Sonntag der THWi als plausibel eingestuft, so dass keine Minderung der Verfügbarkeit durch Brennstoffmangel zu erwarten ist.

Die zusammengefassten Ergebnisse der Funktionszuverlässigkeit je Anbieter und auswertbarer Kalenderwochen sind in der Tab. 4 dargestellt.
Die Funktionszuverlässigkeit konnte während des Projektverlaufs auf mehr als $95 \%$ in der Betriebsphase II verbessert werden und ist vergleichbar mit Werten aus der Literatur, z. B. Serincan (2016) und Kurtz et al. (2015). Brennstoffzellenmodule der Anbieter A und $C$ fielen teilweise auf Grund von geringen Standortlasten aus. Während eines Tests trat dabei meist in einem der zwei BZ-Modulen ein Fehler auf. Obwohl der Standort während der Testzeit durch ein BZ-Modul versorgt wurde, wurde ein solcher Test als nicht funktionsfähig gewertet, da bei einem längeren Stromausfall die Versorgung unter Umständen nicht gewährleistet wäre. Weiterhin gab es ein Softwareproblem mit der Selbsttestroutine, die sich unter Umständen nicht beendete, automatisch abgebrochen wurde und dann wieder neu startete. Einige Softwarefehler wurden während der Projektlaufzeit behoben. Die Funktionszuverlässigkeit kann daher durch weitere Softwareupdates noch signifikant erhöht werden. Auffälligkeiten in den einzelnen Versuchsgruppen waren nicht erkennbar. Das System vom Anbieter B arbeitete sehr zuverlässig mit einer Funktionszuverlässigkeit von 99,89\% über die gesamte Projektlaufzeit. Bei den Untersuchungen zum Betrieb im Winter mit Temperaturen um $0^{\circ} \mathrm{C}$ und im Sommer um die $35^{\circ} \mathrm{C}$, dem Druckgasflaschentausch während des BZ-NEA-Betriebs, der Simulation eines 72 Stunden-Stromausfalls und eines großflächigen Stromausfalls aller Anlagen je Anbieter funktionierten die Brennstoffzellen bis auf die bekannten Fehler ohne Minderung der Verfügbarkeit und Funktionszuverlässigkeit. Eine signifikante Degradation der Brennstoffzellenmodule konnte im Zeitraum des Feldtests nicht beobachtet werden.

Die Berechnungen der Verfügbarkeit und Funktionszuverlässigkeit erfolgten nur für die auswertbaren Kalenderwochen im gesamten Projektverlauf. Es ergaben sich folgende Mittelwerte der auswertbaren Kalenderwochen in

Tab. 3: Übersicht der im Feldtest kumulierten Betriebs- und Ausfallzeiten, sowie der ermittelten BZ-NEA-Verfügbarkeiten aus den auswertbaren Messdaten.

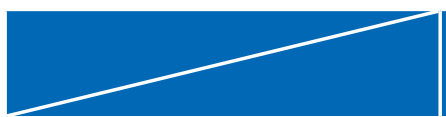

Summe der geplanten Betriebszeiten in $\mathrm{h}$

Anbieter A

Betriebsphase-I Betriebsphase-II Projektverlauf

\section{Anbieter B}

Betriebsphase-I Betriebsphase-II Projektverlauf

Anbieter C

Betriebsphase-I Betriebsphase-II Projektverlauf
898481

131172

362951

494123 
Prozent je Standort: Anbieter A 95,8\%, Anbieter Anbieter B $68 \%$ und Anbieter Anbieter C 97,6\%. Die Messdaten der identischen Monitoringsysteme A und $C$ waren nahezu vollständig auswertbar. Datenlücken entstanden im Wesentlichen durch ein größeres Update der Software. Das Monitoringsystem vom Anbieter B hatte Softwareprobleme im Datenmanagement, so dass historische Messdaten teilweise nicht auswertbar waren. Dies betraf hauptsächlich die Kalenderwochen, in denen keine Tests durchgeführt wurden. Von 1.567 geplanten Tests (Anbieter B) konnten jedoch 1.475 ausgewertet werden. Insgesamt waren je Anbieter $(\mathrm{A} / \mathrm{B} / \mathrm{C})$ 2.037, 1.475 und 1.177 Tests auswertbar.

Der durchschnittliche Untersuchungszeitraum der BZ-NEA betrug 2 Jahre und 1 Monat. Die durchschnittliche BZNEA-Testlaufzeit betrug etwa 1 Stunde je Monat zuzüglich der Selbsttests und sonstigen Notstromfällen. Die akkumulierten ereignisbezogenen BZNEA-Betriebszeiten der auswertbaren Kalenderwochen je Anbieter $(A / B / C)$ betrugen: $2.272 \mathrm{~h}, 2.587 \mathrm{~h}$ und $1.200 \mathrm{~h}$. Auffällig ist Anbieter B mit einer BZNEA-Betriebszeit von $2.587 \mathrm{~h}$, welche sich aus $1.388 \mathrm{~h}$ THWi-Tests, $151 \mathrm{~h}$ sonstigen Notstromfällen und $1.048 \mathrm{~h}$ an Selbsttest zusammensetzt. Im Vergleich zu den anderen Anbietern, wurde im Verlaufe des Projektes ein festes Selbsttestintervall von 14 Tagen mit 45 min Betrieb bei nahezu Nennleistung gefordert. Dieses starre Selbsttestschema hatte einen hohen Wasserstoffverbrauch zur Folge. Über die Projektlaufzeit wurden von den Brennstoffzellen der Anbietern (A/B/C) in Summe $1.619 \mathrm{kWh}, 3.938 \mathrm{kWh}$ und $952 \mathrm{kWh}$ an elektrischer Energie bereitgestellt.

Der BZ-NEA-Verbrauch wurde bei allen Systemen zu ca. $14 \frac{\mathrm{N} l}{\mathrm{~min} \cdot \mathrm{kW}}$ berechnet. Die Berechnung des Brennstoffverbrauchs erfolgte nur näherungsweise, da die Wasserstoffgastemperatur im Feldtest nicht direkt gemessen werden konnte. Es wurde angenommen, dass die Wasserstoffgastemperatur auf Grund der natürlichen Belüftung der Umgebungstemperatur entspricht. Die Herstellerangaben konnten trotz dieser Einschränkung bestätigt werden.

\section{Schlussfolgerungen}

Eine hohe Zuverlässigkeit und Verfügbarkeit der BZ-NEA-Anlagen wurde erfolgreich demonstriert. Entwicklungsbedarf wurde aufgedeckt bei der Detektion von schleichenden Leckagen, der Dichtheit von 300 bar-Wasserstoffarmaturen und den Selbsttestroutinen zur Verfügbarkeitssteigerung. Die Ergebnisse und gesammelten Erfahrungen aus dem Feldtest bestätigen, dass der kommerzielle
Einsatz der Brennstoffzelle als Netzersatzanlage sicherheitsrelevanter Digitalfunkstationen die Praxistauglichkeit erreicht hat.

Tab. 4: Übersicht der ereignisbezogenen Anlagen-Funktionszuverlässigkeiten der jeweiligen Anbieter aus den auswertbaren Messdaten



Summe der Ereignisse

(THWi-Test, Selbsttest und Notstrombetrieb)
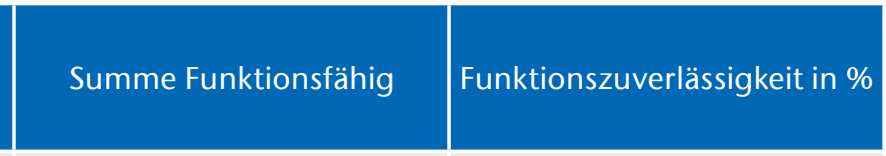

Anbieter A

Betriebsphase-I

Betriebsphase-II

1220

1984

1171

95,98

Projektverlauf

3204

1936

97,58

3107

96,97

\section{Anbieter B}

Betriebsphase-I

Betriebsphase-II

Projektverlauf

\section{Anbieter C}

Betriebsphase-I

Betriebsphase-II

Projektverlauf
1251

2286

3537
99,92

99,87

99,89 


\section{LITERATUR}

Arnold D (ed) (2008) Handbuch Logistik, 3. Aufl. VDIBuch. Springer Berlin Heidelberg, Berlin, Heidelberg. ISBN: 9783540729297

Gagge JP (2008) PEM fuel cells versus diesel generators which solution to pick? In: INTELEC 2008 - 2008 IEEE 30th International Telecommunications Energy Conference, San Diego, CA, pp 1-5. doi: 10.1109/ INTLEC.2008.4664062

Kurtz J, Saur G, Sprik S (2015) Hydrogen Fuel Cell Performance as Telecommunications Backup Power in the United States. Technical Report NREL/TP-540060730, Denver

Kurzel O (2016) Praxisnahe Eignungsuntersuchung von PEM-Brennstoffzellen für Netzersatzanlagen. Bachelorarbeit, Technische Hochschule Wildau

Nordin H, Lindemark B (1999) System reliability, dimensioning and environmental impact of diesel engine generator sets used in telecom applications. In: 1999 Intelec, 9/6/1999 - 9/6/1999, Copenhagen, Denmark.

I E E E, Piscataway, ISBN: 0-7803-5624-1, p 377. doi:

10.1109/INTLEC.1999.794095

Serincan MF (2016) Reliability considerations of a fuel cell backup power system for telecom applications. Journal of Power Sources 309:66-75. doi: 10.1016/j. jpowsour.2016.01.083

Zheng J, Zhang X, Xu P, Gu C, Wu B, Hou Y (2016) Standardized equation for hydrogen gas compressibility factor for fuel consumption applications. International Journal of Hydrogen Energy 41(15):6610-6617. doi: $10.1016 / j$.ijhydene.2016.03.004

\section{AUTOREN}

Prof. Dr. rer. nat. Siegfried Rolle Dr. rer. nat. Lutz B. Giese Dipl--Ing. Michael Jergović

M. Eng. Dipl.-Ing. (FH) Denny Ragusch

Labor für Regenerative Energietechnik

Technische Hochschule Wildau

E-Mail für Korrespondenz:

denny.ragusch@th-wildau.de 